\title{
Regulation of DEK expression by AP-2 $\alpha$ and methylation level of DEK promoter in hepatocellular carcinoma
}

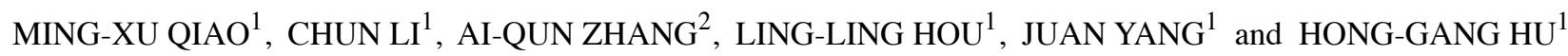 \\ ${ }^{1}$ College of Life Sciences and Bioengineering, School of Science, Beijing Jiaotong University, Beijing 100044; \\ ${ }^{2}$ Institute of Hepatobiliary Surgery, PLA General Hospital, Beijing 100853, P.R. China
}

Received March 5, 2016; Accepted June 23, 2016

DOI: $10.3892 / o r .2016 .4984$

\begin{abstract}
DEK is overexpressed in multiple invasive tumors. However, the transcriptional regulatory mechanism of $D E K$ remains unclear. In the present study, progressive-type truncation assay indicated that CpG2-2 (-167 bp/+35 bp) was the $D E K$ core promoter, whose methylation inhibited DEK expression. Bisulfite genomic sequencing analysis indicated that the methylation levels of the $D E K$ promoter in normal hepatic cells and tissues were higher than those in hepatocellular carcinoma (HCC) cells. TFSEARCH result revealed transcription factor binding sites in $\mathrm{CpG} 2-2$. Among the sites, the AP- $2 \alpha$ binding site showed the most significant methylation difference; hence, AP- $2 \alpha$ is a key transcription factor that regulates DEK expression. Point or deletion mutation of the AP-2 $\alpha$ binding site significantly reduced the promoter activity. Chromatin immunoprecipitation assay demonstrated the binding of AP- $2 \alpha$ to the core promoter. Furthermore, knock down of endogenous AP- $2 \alpha$ downregulated DEK expression, whereas overexpression of AP-2 $\alpha$ upregulated DEK expression. Thus, AP- $2 \alpha$ is an important transcription factor of DEK expression, which is correlated with the methylation level of the $D E K$ core promoter in HCC.
\end{abstract}

\section{Introduction}

Hepatocellular carcinoma (HCC) is one of the most common malignant tumors worldwide; this disease ranks fifth in global morbidity and is the third primary cause of cancer-related mortality (1). Approximately 700,000 new liver cancer cases and deaths are reported annually worldwide, half of which occur in China (2). HCC accounts for $70-85 \%$ of total liver cancer cases (3). Most patients with $\mathrm{HCC}$ are already in middle and advanced stages when properly diagnosed because of lack

Correspondence to: Dr Hong-Gang Hu, College of Life Sciences and Bioengineering, School of Science, Beijing Jiaotong University, Beijing 100044, P.R. China

E-mail: hghu@bjtu.edu.cn

Key words: hepatocellular carcinoma, $D E K$ promoter, methylation, AP- $2 \alpha$, transcriptional regulation of effective diagnostic approaches; although HCC could be treated by excision, therapeutic approaches for this disease are limited, resulting in poor prognosis. Hence, novel specific biomarkers for early diagnosis and prognosis evaluation of HCC must be developed.

Human DEK is a highly abundant chromatin architectural protein (4-7). This protein was initially found in the DEK-CAN fusion protein in acute myeloid leukemia (AML) $(8,9)$. Many studies showed that the DEK protein significantly influences cell cycle and participates in multiple cell functions, such as maintaining the integrity of heterochromatin (10), transcriptional regulation (11), mRNA splicing (12), DNA replication (13) and damage repair and susceptibility (14). The DEK protein is also related to cell apoptosis $(15,16)$. The relationship between $D E K$ and tumor has been increasingly investigated. Studies showed that DEK is overexpressed in multiple malignant human tumors, such as bladder (17), colorectal (18) and gastric cancer (19). In particular, DEK is upregulated in primary HCCs compared with that in matched nonmalignant liver tissues. Thus, DEK is related to poor prognosis and could be used as an independent tumor biomarker for predicting the prognosis of tumors. $D E K$ is also correlated with the occurrence and development of multiple malignant human tumors. However, only few studies reported the overexpression regulatory mechanism of $D E K$ in tumors, particularly the transcriptional regulatory mechanism of $D E K$ in HCC.

DNA methylation has been extensively studied in epigenetics. Researchers reported the presence of abnormal DNA methylation during the formation and development of multiple tumors. DNA methylation occurs when the methyl group $\left(-\mathrm{CH}_{3}\right)$ is transferred from $\mathrm{S}$-adenosylmethionine to the $5^{\prime}$ cytosine of the DNA sequence under the catalysis of DNA methyltransferases (20). Methylation in the genome of mammals mainly occurs at the $\mathrm{CpG}$ site. The $\mathrm{CpG}$ sequence is not uniformly distributed in the genome; the sequence appears less frequently in most regions than other dinucleotide sequences but is more frequent in some regions. A CpG island is a region with high cytosine and guanine contents, sequence length $>200-500 \mathrm{bp}$, GC content $>50 \%$ and $\mathrm{CpG}$ proportion $>0.6$ (21). The $\mathrm{CpG}$ island mainly exists in the gene promoter region and near the first exon region. Among normal human genomes, the $\mathrm{CpG}$ island in genes frequently expressed in cells, such as housekeeping genes, is under non-methylated state. Moreover, inactive genes are normally under high-methylated 
Table I. Primer sequences for luciferase reporter gene vector construction.

\begin{tabular}{llc}
\hline Name & \multicolumn{1}{c}{ Primer sequences (5'-3') } & Length (bp) \\
\hline CpG1 & F-CTAGCTAGCAAAGCATCTGCATAGATGACCTAG & 428 \\
& R-CCCAAGCTTCCTGGAAAAGATGATGAGCAGTC & 345 \\
CpG2-1 & F-CTAGCTAGCTCCAGGAAGCGACCGTGGAAACAATAAAC & \\
& R-CCCAAGCTT TTCAAAATGGCGGTTCGGGAAGGAG & 202 \\
CpG2-2 & F-CTAGCTAGCACTCCAGGCGCAGCCGGGGAGA & 787 \\
CpG3 & R-CCCAAGCTTTTCAAAATGGCGGTTCGGGAAGGAG & \\
& F-CTAGCTAGCAGCATCTGCATAGATGACCTAGAACTC \\
\hline
\end{tabular}

state, which inhibits their expression. $\mathrm{CpG}$ sites on non-CpG islands are under methylated state, which could be preserved stably through cell division (22).

$\mathrm{CpG}$ island in tumor cells manifests methylation abnormality. $\mathrm{CpG}$ island methylation in promoter regions is related to transcriptional silencing; conversely, $\mathrm{CpG}$ site methylation beyond the promoter region is called genosome methylation, which is related to transcriptional activation. Low methylation levels of the entire genome and high methylation levels of the $\mathrm{CpG}$ island in a local region occur simultaneously during tumor formation (23). This abnormal methylation is mainly presented as follows. High methylation levels in the promoter region results in the silencing of cancer suppressor gene, whereas low methylation levels of some oncogenes results in the activation of gene transcription. Given that $D E K$ is a candidate biomarker for tumor diagnosis, the relationship between its overexpression and promoter methylation level, as well as transcriptional regulation of the $D E K$ gene, requires further study to elucidate the regulatory mechanism of $D E K$ overexpression in $\mathrm{HCC}$. The present study aims to provide additional basis for conducting $D E K$ research on HCC treatment.

\section{Materials and methods}

Cell culture. Human HCC cell lines and normal hepatic L02 cells were obtained from the American Type Culture Collection and the Chinese Academy of Sciences, respectively. The cells were cultured in Dulbecco's modified Eagle's medium (DMED; Gibco-Invitrogen, Carlsbad, CA, USA) containing $10 \%$ fetal bovine serum (FBS) in an atmosphere of $5 \% \mathrm{CO}_{2}$ at $37^{\circ} \mathrm{C}$. Normal hepatic tissues were supplied by the PLA General Hospital after receiving informed consent from the patients and obtaining the approval of hospital authorities. The tissues were stored in liquid nitrogen. The institutional ethics committee approved this study.

Western blot analysis. Cells were harvested by trypsinization, counted, and ultrasonically lysed with $200 \mu \mathrm{l}$ of $1 \mathrm{X}$ sodium dodecyl sulfate (SDS) loading buffer per $1 \times 10^{6}$ cells. Protein samples were prepared and loaded at $20 \mu 1$ per lane for SDS-PAGE. The samples were electrotransferred onto nitrocellulose filter membrane, and non-specific binding was blocked in Tris-buffered saline with Tween buffer containing $5 \%$ non-fat dried milk (Yili). Western blot analysis was performed with DEK and AP-2 $\alpha$ (16448-1-AP and 13019-3AP; Proteintech) antibodies, followed by anti-rabbit IgG conjugated with horseradish peroxidase. GAPDH (TA-08; ZSGB-Bio, Beijing, China) was used as loading control. Chemiluminescence signals were quantified using SuperSignal West Femto Maximum Sensitivity Substrate (Pierce).

Real-time quantitative PCR. Total RNA was isolated from cultured cells by using the SV Total RNA Isolation system of RNA extraction kit (Promega, Madison, WI, USA) following the manufacturer's instructions. Total RNA $(1 \mu \mathrm{g})$ was used to synthesize cDNAs by using GoScript ${ }^{\mathrm{TM}}$ Reverse Transcription system (Promega). RT-qPCR reactions using SuperReal PreMix Plus (SYBR-Green; Tiangen Biotech Co., Beijing, China) were performed using Roche LightCycler 480 System. The expression of the gene of interest was normalized to that of $B 2 M$, and relative expression was calculated using comparative $\mathrm{Ct}$ method. PCR primers were as follows: $D E K$, forward, 5'-CAGGCACTGTGTCCTCAT-3' and reverse, 5'-CATTTG GTTCGCTTAGCCT-3'; B2M, forward, 5'-GGCTATCCAGC GTACTCC-3' and reverse, 5'-ACGGCAGGCATACTCA TC-3'.

Reporter gene constructs. Genomic DNA was extracted from eight cell lines and normal hepatic tissues by using QIAamp DNA Mini kit (Qiagen, Hilden, Germany) following the manufacturer's instructions. $D E K$ promoter $\mathrm{CpG}$ island region was truncated into four fragments, namely, CpG1 (-732 bp/-305 bp), CpG2-1 (-310 bp/+35 bp), CpG2-2 (-167 bp/+35 bp) and CpG3 (-730 bp/+57 bp). Four pairs of primers for PCR amplification were designed by Primer Premier 5.0 software (Table I). The amplification products were cloned into the pGL3-Basic luciferase reporter gene vector (Promega) and named as pGL3$D E K / \mathrm{CpG} 1, \mathrm{pGL} 3-D E K / \mathrm{CpG} 2-1$, pGL3-DEK/CpG2-2 and pGL3-DEK/CpG3. The linear plasmids digested by HindIII of the four recombinant plasmids were treated with Sss I methylation enzyme, and their methylation status was identified using BstUI (methylation-sensitive restriction enzyme). The completely methylated linear plasmids were digested by NheI to obtain the full methylation of the four target fragments, which were cloned into the pGL3-Basic vector. The resulting vectors were called pGL3-Basic/CpG1 (Me), pGL3-Basic/ CpG2-1 (Me), pGL3-Basic/CpG2-2 (Me) and pGL3-Basic/ CpG3 (Me). 
Table II. Primer sequences for BSP.

Name

Primer sequences $\left(5^{\prime}-3^{\prime}\right)$

Length (bp)

C1

F-TTTTATATTTATAGGGGTGTAAATTTATGT

$\mathrm{C} 2$ R-CTTCCTAAAAAAAATAATAAACAATCCC

Transfection and luciferase assays. The cells were plated in 96-well or 24-well plates, grown to $60-80 \%$ confluence, and transfected with small-interfering RNA (siRNA) or plasmids by using Lipofectamine ${ }^{\circledR} 2000$ reagent (Invitrogen) according to the manufacturer's instructions. Cells transfected with luciferase constructs were harvested and lysed in Passive lysis buffer (Promega) after $48 \mathrm{~h}$. The cell lysates were analyzed for firefly and Renilla luciferase activity by using the DualLuciferase reporter assay system (Promega) as recommended by the manufacturer. Firefly luciferase activity was normalized to Renilla luciferase activity as internal control.

Bisulfite genomic sequencing. The genomic DNA of eight HCC cell lines and normal hepatic tissue was treated with sodium bisulfite by EpiTect ${ }^{\circledR}$ Fast Bisulfite Conversion DNA kit (Qiagen). PCR amplification fragment should not be too long $(<500 \mathrm{bp})$ because the treated genomic DNA becomes unstable and easy to break. Thus, we divided the $\mathrm{CpG}$ island sequence into two short segments to amplify 294 and $381 \mathrm{bp}$ in the $D E K$ promoter $-595 \mathrm{bp} /-302 \mathrm{bp}$ and $-329 \mathrm{bp} /+52 \mathrm{bp}$, respectively. The corresponding segments were named $\mathrm{Cl}$ and $\mathrm{C} 2$. We then designed two pairs of primers (Table II) for the treated $\mathrm{C} 1$ and $\mathrm{C} 2$ for bisulfite sequencing PCR (BSP). BSP was performed in 40 cycles of $98^{\circ} \mathrm{C}$ for $10 \mathrm{sec}, 55^{\circ} \mathrm{C}$ for $30 \mathrm{sec}$, and $72^{\circ} \mathrm{C}$ for $30 \mathrm{sec}$. Finally, the PCR products were cloned into the pMD18-T vector (Takara). At least 10 clones were randomly selected for bisulfite genome sequencing (BGS) to analyze the methylation status of the $D E K$ promoter $\mathrm{CpG}$ island.

Prediction of transcription factor binding sites of DEK promoter $\mathrm{Cp} G$ island region. Bioinformatics methods were used to predict the $\mathrm{CpG}$ island location in the $D E K$ promoter region near the sequence from -1000 to +400 bp range by MethPrimer online software (http://www.urogene.org/cgi-bin/ methprimer/methprimer.cgi). The transcription factor binding sites of $D E K$ promoter $\mathrm{CpG}$ island region were predicted by TFSEARCH online software (http://www.cbrc.jp/research/db/ TFSEARCH.html).

Point and deletion mutation assays. We analyzed point and deletion mutation of the binding site 'GCCCGCGGC' of the transcription factor $\mathrm{AP}-2 \alpha$ in the $\mathrm{CpG} 2-2$ region. Point mutation is the substitution of the underlined consensus sequence 'GCCCGCGGC' into 'TAACGCGGC'. Deletion mutation includes the deletion of the underlined part of 'GCCCGCGGC' and the removal of all 'GCCCGCGGC'. The three kinds of mutational $\mathrm{CpG}$-2 fragments, namely, pGL3-basic/PM, pGL3-basic/FM and pGL3-basic/AM, were cloned into the
pGL3-Basic vector (Promega). Finally, HepG2 cells were transfected with the plasmids by using Lipofectamine ${ }^{\circledR} 2000$ reagent (Invitrogen) according to the manufacturer's instructions. Blank plasmid pGL3-basic and pGL3-DEK/CpG2-2 were used as negative and positive controls. Luciferase activity was assayed after $24 \mathrm{~h}$.

Chromatin immunoprecipitation assay. Chromatin immunoprecipitation (ChIP) assay was performed with EpiQuik ChIP kit (P-2002-1; EpiGentek, Farmingdale, NY, USA) following the manufacturer's instructions. A total of $1 \times 10^{6}$ HepG2 cells were fixed in $1 \%$ formaldehyde at room temperature for $10 \mathrm{~min}$. The cells were then lysed and chromatin was sheared by sonication. The DNA-protein complex was immunoprecipitated with anti-RNA Pol II antibody (P-2002-1; EpiGentek), anti-IgG antibody (P-2002-1; EpiGentek), and anti-AP-2 $\alpha$ antibody (13019-3-AP; Proteintech). After reverse crosslinking and DNA purification, DNA from input (1:20 diluted) or immunoprecipitated samples were assayed by PCR. The products were separated by $3 \%$ agarose gel electrophoresis. The primers for the $D E K$ promoter were (forward) 5'-GCGA CCGTGGAAACAATAAACA-3' and (reverse) 5'-TGCCTCC GCGGGAAGCTC-3'. Primers for the positive control GAPDH were (forward) 5'-ACGTAGCTCAGGCCTCAAGA-3' and (reverse) 5'-GCGGGCTCAATTTATAGAAAC-3' (P-2002-1; EpiGentek).

Overexpression and small-interfering RNA interference assays of transcription factor AP- $2 \alpha$. AP- $2 \alpha$ expression plasmid was constructed according to the CDS coding region of TFAP- $2 \alpha$ (NM_001032280.2) in the National Center for Biotechnology Information website (www.ncbi.nlm.nih.gov/). The modified CDS of TFAP- $2 \alpha$ was inserted with the His tag sequence 'CATCACCATCACCATCAC' behind the transcription start site 'ATG' and added with two restriction enzyme cutting sites on both ends, namely, 5'-end EcoRI 'GAATTC' and 3'-end HindIII 'TTCGAA'. The modified CDS was then cloned into the pcDNA3.1 (-) vector (Invitrogen) and named pcDNA3.1 (-)-hAP2. The vector was transfected into HepG2 cells, and the expression levels of $A P-2 \alpha$ and $D E K$ were assayed. In addition, $A P-2 \alpha$ siRNA interference sequences and siRNA-negative control (NC) (Table III) used in this study were designed and synthesized by GenePharma. Equimolar amounts of three oligonucleotides specific for $A P-2 \alpha$ were combined before transfecting 7721 cells. Blank control, NC, and the experiment group were set up for the transfection experiment. Total RNA was extracted using SV Total RNA Isolation system of RNA extraction kit (Promega) after $24 \mathrm{~h}$ of transfection. Total protein was extracted after transfection of $48 \mathrm{~h}$. Finally, the mRNA and 
Table III. AP-2 $\alpha$ small-interfering RNA interference sequences.

\begin{tabular}{lcc}
\hline Name & Sense $\left(5^{\prime}-3^{\prime}\right)$ & Antisense $\left(5^{\prime}-3^{\prime}\right)$ \\
\hline siRNA-1 & CCAGAUCAAACUGUAAUUAdTdT & UAAUUACAGUUUGAUCUGGdTdT \\
siRNA-2 & GGAAGAUCUUUAAGAGAAAdTdT & UUUCUCUUAAAGAUCUUCCdTdT \\
siRNA-3 & CCUGCUCACAUCACUAGUAdTdT & UACUAGUGAUGUGAGCAGGdTdT \\
siRNA-NC & UUCUCCGAACGUGUCACGUdTdT & ACGUGACACGUUCGGAGAAdTdT \\
\hline
\end{tabular}

Table IV. Primer sequences for reverse transcription-PCR.

\begin{tabular}{llc}
\hline Name & \multicolumn{1}{c}{ Primer sequences (5'-3') } & Length (bp) \\
\hline$D E K$ & F-CAGGCACTGTGTCCTCAT & 316 \\
& R-CATTTGGTTCGCTTAGCCT & \\
$A P-2 \alpha$ & F-CGTCTCCGCCATCCCTATTA & 364 \\
& R-GGTTTCGCACACGTACCCAA & \\
$B 2 M$ & F-GGCTATCCAGCGTACTCC & 247 \\
& R-ACGGCAGGCATACTCATC & \\
\hline
\end{tabular}

protein expression levels of $A P-2 \alpha$ and $D E K$ were investigated by RT-PCR and western blot analyses. The primer sequences for $A P-2 \alpha$ and $D E K$ are shown in Table IV.

Statistical analysis. Statistical analysis was performed using SPSS software. Results were analyzed by Student's t-test, and differences were considered statistically significant at $\mathrm{P}<0.05$. All in vitro experiments were performed in triplicate, and the average result is reported. Error bars depict standard error.

\section{Results}

$D E K$ is overexpressed in HCC. Fluorescent quantitative PCR and western blot analyses were performed to determine the mRNA and protein expression levels of DEK in HCC cell lines, respectively. The results showed that $D E K$ mRNA and protein were overexpressed in all HCC cell lines compared with those in normal hepatic cell line L02 and tissues (Fig. 1). Gel-Pro Analyzer was used to quantify the western blotting results, and two-tailed Student's t-test indicated that $D E K$ mRNA and protein expression levels in the seven HCC cell lines $(\mathrm{P}<0.05$ or $\mathrm{P}<0.01)$ significantly differed from those in L02 and normal hepatic tissues. Among the cell lines, 7402 and 7721 HCC cells exhibited the highest DEK expression level. DEK was found to be overexpressed in HCC.

CpG2-2 (-167 bp/+35 bp) is the core promoter region of $D E K$. Sequences near the $D E K$ promoter region within $-1000 \mathrm{bp} /+400 \mathrm{bp}$ were analyzed through bioinformatics methods with the MethPrimer online software. Potential $\mathrm{CpG}$ island was located within $-436 \mathrm{bp} /+345 \mathrm{bp}$ (Fig. 2A). The extension of the $D E K$ core promoter was then identified. A series of reporter gene constructs was obtained by progressive-type truncation strategy, transfected into HepG2 cells, and then analyzed with the Dual-Luciferase reporter assay system. The results
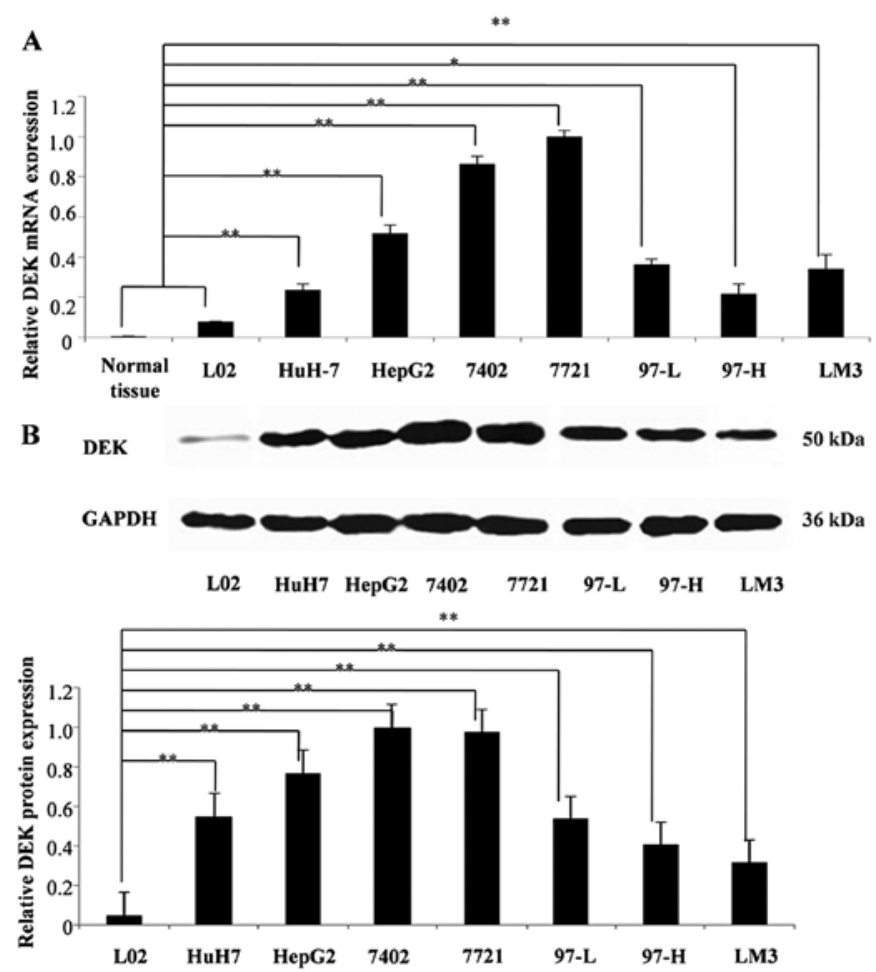

Figure 1. Analysis of DEK expression level by real-time quantitative PCR (RT-qPCR) and western blot analyses. (A) $D E K$ mRNA level in hepatocellular carcinoma (HCC) cell lines was assayed by RT-qPCR, with normal hepatic cell L02 and normal tissues as controls. (B) DEK protein level was detected by western blotting and gray scanning analysis of Gel-Pro Analyzer software, with normal hepatic cell L02 as control. Data are shown as averages of three independent experiments. ${ }^{*} \mathrm{P}<0.05,{ }^{* *} \mathrm{P}<0.01$.

demonstrated that among the constructs without methylation in vitro, the activities of pGL3-DEK/CpG3, pGL3-DEK/CpG2-1, and pGL3-DEK/CpG2-2 were higher than those of pGL3-DEK/ $\mathrm{CpG} 1$ compared with the controls $(\mathrm{P}<0.01)$. However, the activities of pGL3-DEK/CpG2-1 and pGL3-DEK/CpG2-2 were not significantly different from that of pGL3-DEK/CpG3 ( $\mathrm{P}>0.05)$. Further analysis showed that pGL3-DEK/CpG2-2 reserved most of the activity of pGL3-DEK/CpG2-1 (P>0.05) (Fig. 2B); hence, $\mathrm{CpG} 2-2(-167 \mathrm{bp} /+35 \mathrm{bp})$ is the minimum core promoter region of the $D E K$ gene. In addition, no activity was found in HepG2 cells for constructs methylated in vitro compared with that in NC. This result illustrates that hypermethylation of the $D E K$ promoter inhibited its activity in HCC.

DEK promoter in HCC is commonly under low-methylation state. BSP and BGS were performed using genomic DNA of eight HCC cell lines and normal hepatic tissues to investigate 


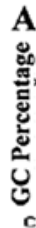

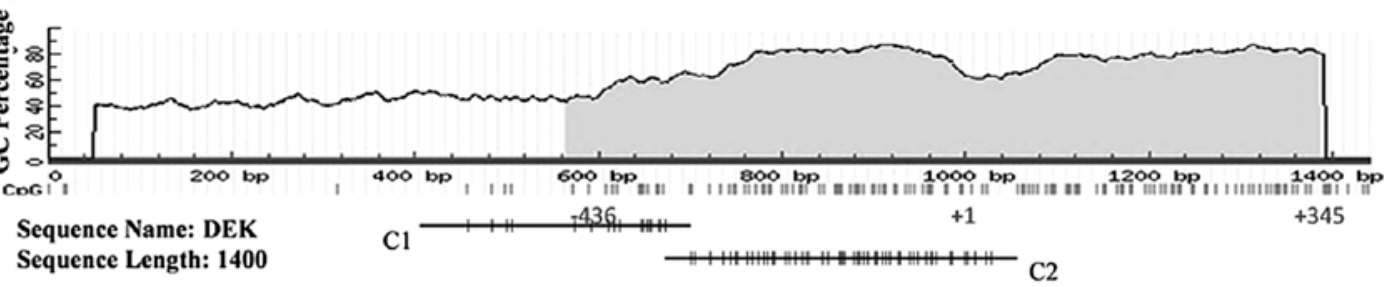

CpG island prediction results

(Criteria used: island size $>100$, GC Percent $>50.0,0 \mathrm{bs} / \mathrm{Exp}>0.6$ )

$1 \mathrm{CpG}$ island(s) were found in your sequence

$\begin{array}{llr} & \text { Size } & \text { (Start - End) } \\ \text { Island } 1 & \mathbf{7 8 1} \text { bp } & (565-1345)\end{array}$

B
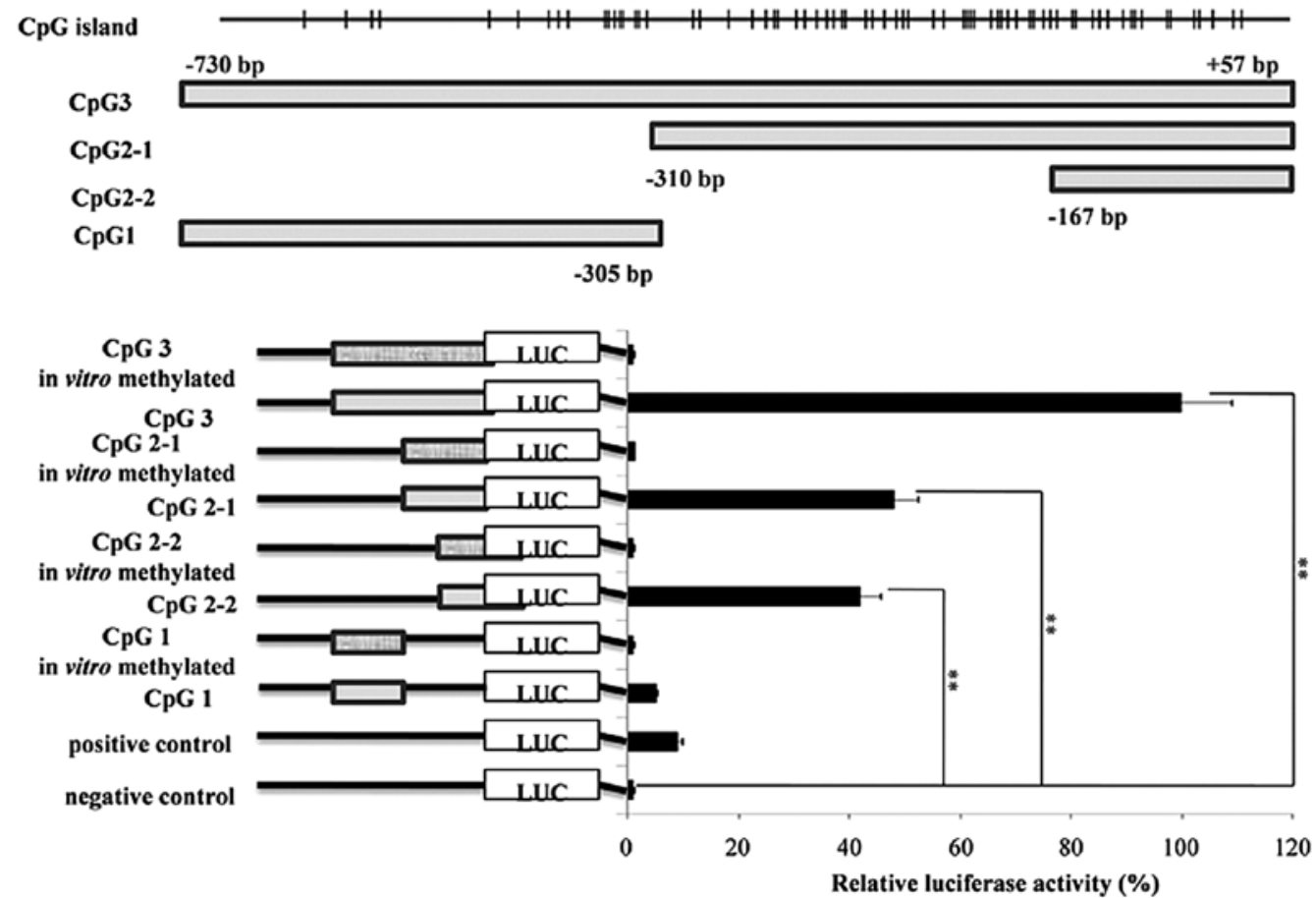

Figure 2. Identification of the core promoter of human $D E K$ in HepG2 cells and effect of DNA methylation on promoter activity. (A) The CpG island of $D E K$ promoter region near the sequence from $-1000 \mathrm{bp}$ to $+400 \mathrm{bp}$ range predicted by MethPrimer online software was located in $-436 \mathrm{bp} /+345 \mathrm{bp}$ region. In the schematic structure of the $\mathrm{CpG}$ island, short vertical lines represent $\mathrm{CpG}$ sites, and '+1' denotes transcription start site. (B) Identification of the core promoter of human $D E K$ by truncation analysis. CpG island region was truncated into four fragments, namely, CpG1 (-732 bp/-305 bp), CpG2-1 (-310 bp/ $+35 \mathrm{bp}), \mathrm{CpG} 2-2$ $(-167 \mathrm{bp} /+35 \mathrm{bp})$, and CpG3 (-730 bp/+57 bp). HepG2 cells were transiently transfected with the luciferase reporter constructs. Reporter gene activity was assayed by the Dual-Luciferase reporter assay system, and pGL3-basic vector was used as negative control. Relative firefly luciferase activity was calculated as the average of three independent transfections normalized to Renilla control activity $\left({ }^{* *} \mathrm{P}<0.01\right)$.

whether the methylation level of the $D E K$ promoter is related to its overexpression in HCC. We encoded the sequencing results into QUMA online software for comparative analysis. The atlas shown in Fig. 3A illustrates the detailed $\mathrm{CpG}$ methylation sites of $D E K$ promoters. The results proved that the methylation levels of $D E K$ promoters in normal hepatic tissues and normal hepatic L02 were significantly higher than those in the HCC cell lines; moreover, methylation sites were mostly located in the $\mathrm{CpG} 2-2$ region. We then calculated the total ratio of methylation sites and plotted the methylation ratio histogram (Fig. 3B) to investigate the methylation level of $D E K$ promoters in all cell lines and tissues. The result showed that DEK promoters in HCC were commonly under low-methylation state compared with those in normal cells and tissues; hence, $D E K$ overexpression in $\mathrm{HCC}$ was correlated with the low-methylation level of the promoters.
Transcription factor binding sites of DEK core promoter prediction. We speculated that the $D E K$ core promoter region may contain important transcription factor binding sites that play pivotal roles in maintaining the basal transcriptional activity of $D E K$. Hence, we analyzed the core promoter region CpG2-2 (-167 bp/+35 bp) by using TFSEARCH online software. The results showed that DNA binding sites for four transcription factors, namely, nuclear factor Y (NF-Y), AP-2 $\alpha$, E2F and Ying Yang 1 (YY1), were found in the CpG2-2 region. According to the BGS results (Fig. 3A), transcription factor binding sites containing $\mathrm{CpG}$ methylation sites in normal hepatic cells and tissues were screened; these sites included AP-2 $\alpha$, E2F and YY1 (Fig. 4A). Among these sites, the binding site forAP- $2 \alpha$ presented the most significant methylation difference compared with that in $\mathrm{HCC}$ and was located in sites no. 45 and $46 \mathrm{CpG}$ (Fig. 3A). The results suggested 


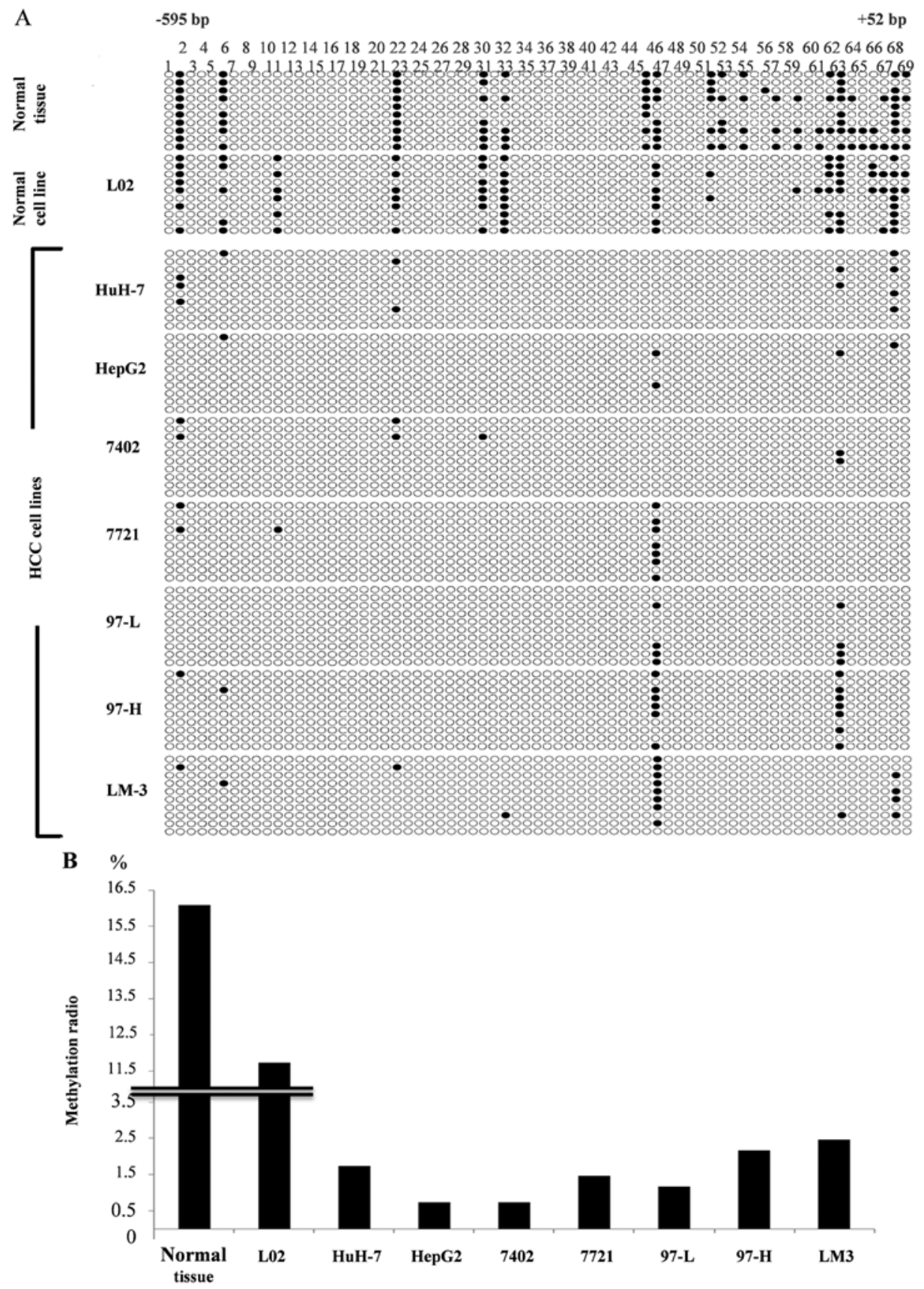

Figure 3. Methylation analysis of $D E K$ promoter in $\mathrm{HCC}$ cells, L02 and normal hepatic tissues. (A) Representative high-resolution analysis of the methylation status of individual $\mathrm{CpG}$ sites in the $D E K$ promoter $(\mathrm{C} 1$ and $\mathrm{C} 2,-595 \mathrm{bp} /+52 \mathrm{bp})$ by bisulfite genomic sequencing in $\mathrm{HCC}$ cell lines and normal hepatic cell L02 and tissues. At least 10 clones were randomly chosen and sequenced. Each circle corresponds to a single $\mathrm{CpG}$ site. Methylated sites are indicated by filled dark circles, whereas unmethylated sites by empty white ones $(\bullet$, methylatedCpG site; $\circ$, unmethylatedCpG site) from -595 bp to +52 bp in turn numbering 1 , $2,3, \ldots 68$ and 69 . (B) The methylation ratio of every cell and tissue was calculated, and methylation ratio histogram was plotted.

that $D E K$ overexpression in HCC was correlated with the demethylation of the AP-2 $\alpha$ transcription factor binding site. In addition, transcription factors NF-Y, E2F and YY1 were previously reported to regulate DEK expression $(24,25)$. We will focus on the regulation effect of the transcription factor AP-2 $\alpha$ for DEK expression in further studies.

AP-2 $\alpha$ binding site plays a dominant role in maintaining DEK core promoter activity. We evaluated the effect of the predicted AP-2 $\alpha$ transcription factor binding site on DEK core promoter activity. We performed point and deletion mutations in the wild-type plasmid pGL3-DEK/CpG2-2 and transfected the constructs into HepG2 cells. Luciferase activities were determined after $24 \mathrm{~h}$. The results showed that $D E K$ core promoter activity was significantly reduced after point and deletion mutations of the AP- $2 \alpha$ transcription factor binding site $(\mathrm{P}<0.01)$. In particular, all deletion mutation-type pGL3-basic/AM led to $\sim 60 \%$ reduction in DEK core promoter activity compared with that in the wild-type pGL3-DEK/CpG2-2 (Fig. 4B). This result indicated that the AP- $2 \alpha$ transcription factor binding site plays a dominant role in maintaining the transcription activity of the $D E K$ core promoter. We also predict that the newly found transcription factor AP- $2 \alpha$ may significantly regulate DEK expression. 
A

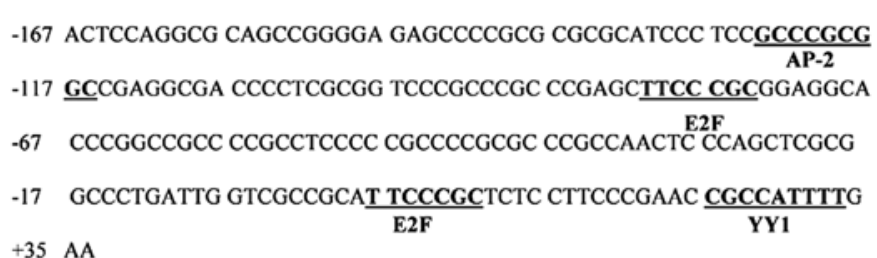

B

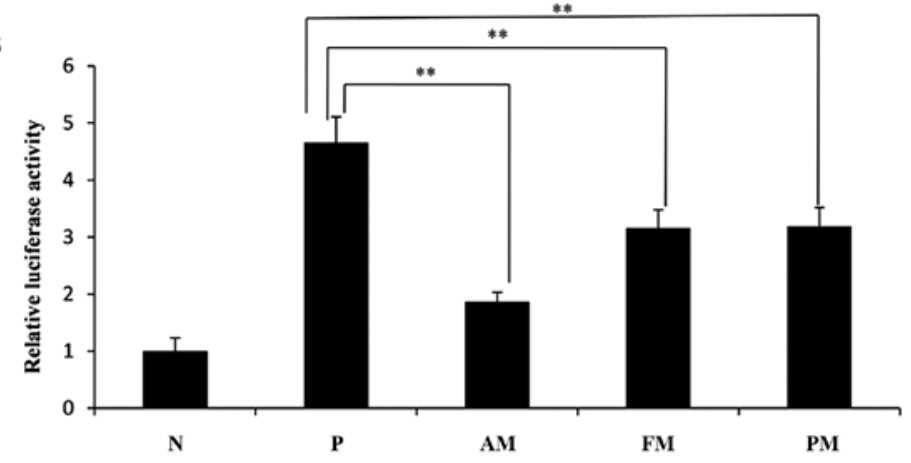

C

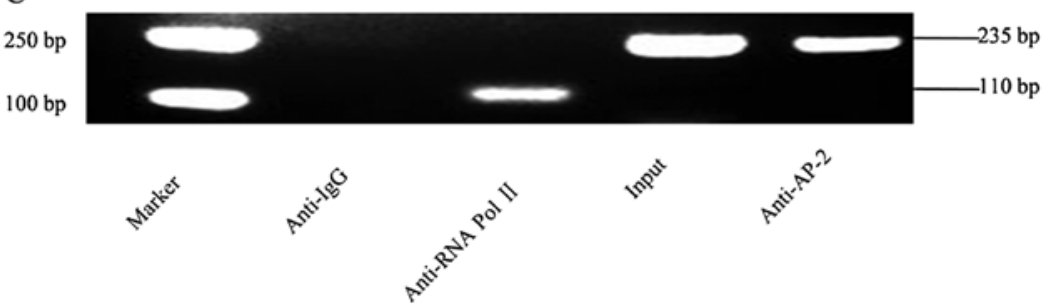

Figure 4. Characterization of transcription factor binding sites. (A) Nucleotide sequence of the $D E K$ core promoter was located from -167 bp to +35 bp. Putative transcription factor binding sites were underlined. (B) Mutation analysis of putative transcription factor AP-2 $\alpha$ binding site. HepG2 cells were transfected with the pGL3-basic vector, the wild plasmid pGL3-DEK/CpG2-2, three nucleotide substitution mutant variant (pGL3-basic/PM), six site-deletion mutant variant (pGL3-basic/FM), and all site-deletion mutant variant (pGL3-basic/AM). Luciferase activity was measured after 24 h. The negative control was normalized to 1, and others were compared with the normalized level. N, negative control; P, positive control; PM, point mutation; FM, fraction deletion; AM, all deletion. Transfections were performed in triplicate for each experiment $\left({ }^{* *} \mathrm{P}<0.01\right)$. (C) The binding of AP- $\alpha \alpha$ to the DEK core promoter in vivo using Chromatin immunoprecipitation assay. The nucleoprotein complex from HepG2 cells was immunoprecipitated with anti-IgG, anti-RNA Pol II and anti-AP-2 $\alpha$ antibodies, respectively. Then, the precipitated and purified DNA fragments were subjected to PCR. Anti-IgG antibody (negative control) failed to precipitate any proteinDNA complex in vivo; anti-RNA Pol II antibody (positive control) precipitated the RNA Pol II bound to the GAPDH promoter and the PCR amplified products were $110 \mathrm{bp}$ (RNA Pol II is considered to be enriched in the GAPDH gene promoter); anti-AP-2 $\alpha$ antibody precipitated AP-2 $\alpha$ proteins bound to the $D E K$ core promoter which contains the predicted AP-2 $\alpha$ binding sites 'GCCCGCGGC' in vivo and its PCR amplified products were 235 bp. The input served as the internal reference and measure the efficiency of ChIP.

Transcription factor AP-2 $\alpha$ binds to the DEK core promoter in vivo. AP- $2 \alpha$ is a transcription factor that binds to the promoter regions of its target genes, which contain the binding sites 'GCCCGCGGC', and mediates the transcription of the target genes. We performed ChIP assay to validate whether AP-2 $\alpha$ interacts with the $D E K$ core promoter in vivo. The nucleoprotein complex prepared from HepG2 cells was immunoprecipitated with anti-RNA Pol II, anti-IgG and antiAP- $2 \alpha$ antibodies. The precipitated DNA was subjected to PCR with primers flanking the region containing the AP- $2 \alpha$ binding site. The PCR results showed that anti-AP- $2 \alpha$ antibody precipitated proteins were bound in vivo to the amplified sequence of the DEK promoter; by contrast, non-specific $\operatorname{IgG}$ antibody (NC antibody) failed to precipitate proteins bound in vivo in this sequence (Fig. $4 \mathrm{C}$ ). Thus, the transcription factor AP- $2 \alpha$ could bind to the $D E K$ core promoter region in vivo.

Transcription factor AP-2 $\alpha$ upregulates the expression level of the DEK gene. AP- $2 \alpha$ siRNA or AP- $2 \alpha$ expression vector pcDNA3.1 (-)-hAP2 was transfected into 7721 or HepG2 cells to determine the role of AP- $2 \alpha$ in regulating DEK expression. $D E K$ mRNA and protein levels were detected by RT-PCR and western blot analyses, respectively. The results illustrated that siRNA mediated 53\% knockdown of AP- $2 \alpha$ mRNA and $80 \%$ knockdown of the $A P-2 \alpha$ protein, resulting in reduction in $D E K$ mRNA level by $51 \%$ and DEK protein level by $63 \%$ (Fig. 5A and B). By contrast, overexpression of $A P-2 \alpha$ mRNA by 1.9-fold and AP- $2 \alpha$ protein by 2.0 -fold led to 2.5 -fold increase in $D E K$ mRNA level and a substantial increase of $90 \%$ in the DEK protein level compared with those in the blank control (Fig. 5C and D). Thus, we conclude that the expression of DEK was mediated by the transcription factor AP- $2 \alpha$.

\section{Discussion}

We performed RT-qPCR and western blot analyses to verify that DEK was really overexpressed in HCC. The mRNA and protein expression levels of DEK increased, which were identical with the results reported in previous studies (26-28). However, minimal information is available on the regulation of DEK expression. The overexpression of DEK in HCC was 
A

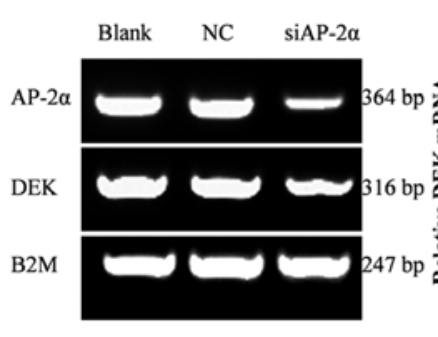

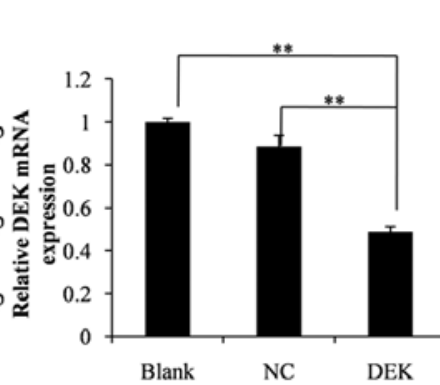

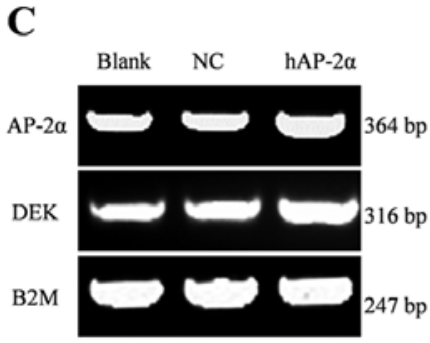

B

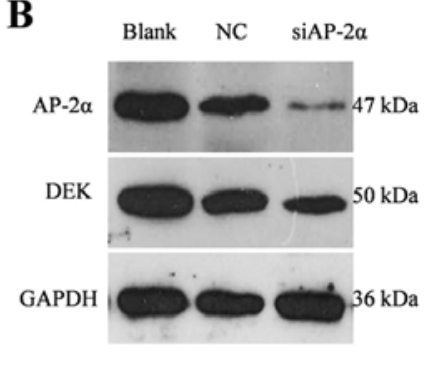

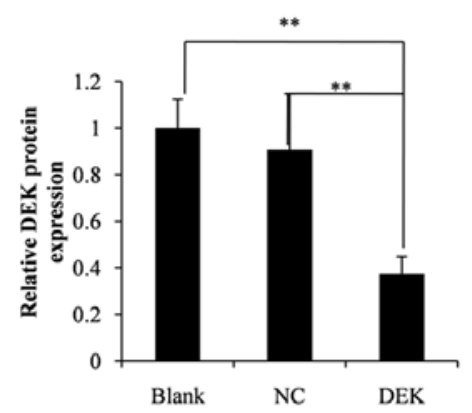

D
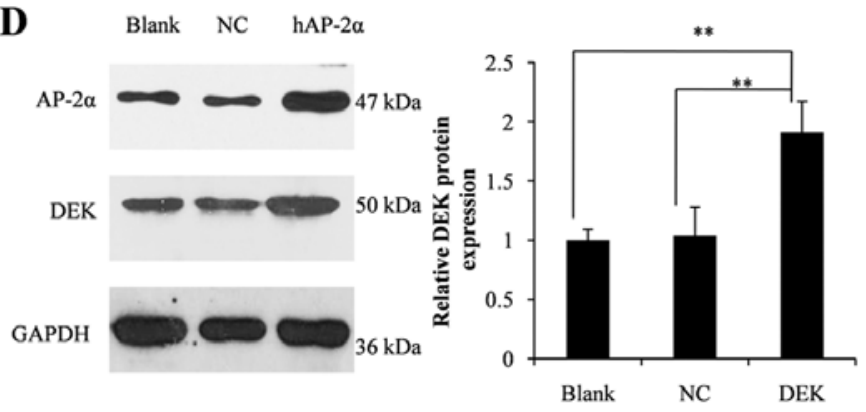

Figure 5. AP-2 $\alpha$ regulation of DEK expression in HCC cells. HepG2 cells were transiently transfected with AP-2 $\alpha$ siRNA and negative control siRNA. A total of 7721 cells were transiently transfected with pcDNA3.1 (-) empty vector and pcDNA3.1 (-)-hAP2. (A and C) DEK mRNA was determined through RT-PCR $24 \mathrm{~h}$ after transfection, and the gray scanning results of BandScan were normalized to B2M. Data are representative of three independent experiments $\left({ }^{* *} \mathrm{P}<0.01\right)$. (B and D) Interference efficiency, overexpression efficiency, and DEK protein expression were analyzed by western blotting with anti-AP-2 $\alpha$, antiDEK and anti-GAPDH antibodies. Gray scanning results of BandScan were normalized to GAPDH. The blank control was normalized to 1, and others were compared relative to the normalized level. Blank, blank control; $\mathrm{NC}$, negative control. Data are representative of three independent experiments $\left({ }^{* *} \mathrm{P}<0.01\right)$.

initially reported to be of S-phase dependency and could exert carcinogenic effect by inhibiting p53 activity (29). Previous studies showed that the proportion of $D E K$ mRNA overexpression in HCC tissues was rather high, which may be related to the conversion of normal hepatic tissues into HCC (26). In addition, reports suggested that $D E K$, as an independent risk factor in HCC, promoted tumor invasiveness, resulting in the poor prognosis of HCC (27). Hence, the abnormal overexpression of DEK in tumors would probably become a biomarker for early diagnosis or vicious transformation. Nevertheless, studies on the transcriptional regulatory mechanism of the abnormal overexpression of DEK in tumors were not comprehensive. The $D E K$ promoter contains transcription factor binding sites bound by transcription factors E2F, YY1, ER $\alpha, N F-Y$ and c-myc. E2F and YY1 were bound to the sequences near the $D E K$ transcription start site, which induced the formation of a transcriptional initiation complex to activate the relevant passageways and boost tumor formation.

Several studies on the transcriptional regulatory mechanism of genes in tumors revealed that decreased methylation levels of oncogenes are common in human tumors. Methylation level is inversely correlated with gene expression. The low-methylation level of many genes in tumor tissues occurs in the promoter region. The low-methylation level of LINE-1 in chronic granulocytic leukemia could initiate its sense transcription and antisense transcription of $c$-MET (30). Numerous activated and amplified $c$-fos genes were found in ovarian cancer tissues of mice, primarily because of the lowmethylation level of the $c$-fos gene (31). Proto-oncogene $c$-myc was also under the low-methylation state in tumor tissues, which led to significant upregulation of gene expression and then accelerated cell malignant proliferation, resulting in tumor occurrence (32). Additional studies reported that the promoter region of estrogen receptor (ER) regulatory gene $p S 2$ in breast cancer was lowly methylated, which is significantly related to the upregulation of ER (33). Moreover, the low-methylation level of multiple genes often occurs in the same kind of tumor. For example, genes, such as claudin 4, LCN2,TFF2,S100A4 and mesothelin, are all lowly methylated in pancreatic cancer (34).

The present study revealed that the functional minimal core promoter CpG2-2 is located within the 202 bp region at position $-167 \mathrm{bp} /+35 \mathrm{bp}$ relative to the transcription start site by Dual-Luciferase reporter assay. BGS was performed to evaluate the effect of methylation level on $D E K$ promoter transcriptional activity. The results suggested that $D E K$ promoter in HCC cell lines was commonly under low-methylation state compared with normal hepatic tissues and normal hepatic cell line L02. Moreover, methylation sites focused on CpG2-2 region, which indicated that DEK overexpression in $\mathrm{HCC}$ is related to the low-methylation level of the core promoter. Hypermethylation exerted a remarkable inhibiting effect on its transcriptional activity. According to the BGS results, AP- $2 \alpha$ transcription factor binding site predicted by TFSEARCH online software was screened as the most hypermethylated site in normal hepatic cells and tissues. Furthermore, we verified whether transcription factor AP- $2 \alpha$ regulates DEK expression. We performed point and deletion mutations of the AP- $2 \alpha$ DNA binding site and ChIP assays. The results demonstrated that the AP-2 $\alpha$ binding site was crucial for the transcription activity of the $D E K$ core promoter, and AP- $2 \alpha$ transcription factor could bind to the $D E K$ core promoter region in vivo. Knocking down endogenous AP-2 $\alpha$ led to reduction in DEK expression. Conversely, overexpression of AP-2 $\alpha$ upregulated 
DEK expression. The transcription factor AP-2 $\alpha$ regulates DEK expression by directly binding to the AP- $2 \alpha$ binding site.

In summary, the present study reveals that the $D E K$ core promoter is located in the $-167 \mathrm{bp} /+35 \mathrm{bp}$ region. DEK overexpression in $\mathrm{HCC}$ is regulated by the transcription factor AP- $2 \alpha$ and closely correlated with the methylation level of the core promoter. This study further reveals the transcriptional regulatory mechanism of DEK overexpression in $\mathrm{HCC}$ and provides additional basis for early diagnosis, prognosis judgment, and genetic therapy in $\mathrm{HCC}$.

\section{Acknowledgements}

We are grateful to the PLA General Hospital for providing the normal hepatic tissue sample. The present study was supported by the National Natural Science Foundation of China (grant no. 81201762).

\section{References}

1. Raza A and Sood GK: Hepatocellular carcinoma review: Current treatment, and evidence-based medicine. World J Gastroenterol 20: 4115-4127, 2014.

2. Ferlay J, Shin HR, Bray F, Forman D, Mathers C and Parkin DM: Estimates of worldwide burden of cancer in 2008: GLOBOCAN 2008. Int J Cancer 127: 2893-2917, 2010.

3. Perz JF, Armstrong GL, Farrington LA, Hutin YJ and Bell BP. The contributions of hepatitis $B$ virus and hepatitis $C$ virus infections to cirrhosis and primary liver cancer worldwide. J Hepatol 45: 529-538, 2006

4. Hu HG, Scholten I, Gruss C and Knippers R: The distribution of the DEK protein in mammalian chromatin. Biochem Biophys Res Commun 358: 1008-1014, 2007.

5. Waldmann T, Baack M, Richter N and Gruss C: Structurespecific binding of the proto-oncogene protein DEK to DNA. Nucleic Acids Res 31: 7003-7010, 2003.

6. Waldmann T, Eckerich C, Baack M and Gruss C: The ubiquitous chromatin protein DEK alters the structure of DNA by introducing positive supercoils. J Biol Chem 277: 24988-24994, 2002.

7. Waldmann T, Scholten I, Kappes F, Hu HG and Knippers R: The DEK protein: an abundant and ubiquitous constituent of mammalian chromatin. Gene 343: 1-9, 2004.

8. von Lindern M, Fornerod M, van Baal S, Jaegle M, de Wit T, Buijs A and Grosveld G: The translocation (6;9), associated with a specific subtype of acute myeloid leukemia, results in the fusion of two genes, dek and can, and the expression of a chimeric, leukemia-specific dek-can mRNA. Mol Cell Biol 12: 1687-1697, 1992.

9. Soekarman D, von Lindern M, Daenen S, de Jong B, Fonatsch C, Heinze B, Bartram C, Hagemeijer A and Grosveld G: The translocation $(6 ; 9)(\mathrm{p} 23 ; \mathrm{q} 34)$ shows consistent rearrangement of two genes and defines a myeloproliferative disorder with specific clinical features. Blood 79: 2990-2997, 1992.

10. Kappes F, Waldmann T, Mathew V, Yu J, Zhang L, Khodadoust MS, Chinnaiyan AM, Luger K, Erhardt S, Schneider $\mathrm{R}$, et al: The DEK oncoprotein is a $\mathrm{Su}(\mathrm{var})$ that is essential to heterochromatin integrity. Genes Dev 25: 673-678, 2011.

11. Fu GK, Grosveld G and Markovitz DM: DEK, an autoantigen involved in a chromosomal translocation in acute myelogenous leukemia, binds to the HIV-2 enhancer. Proc Natl Acad Sci USA 94: 1811-1815, 1997.

12. McGarvey T, Rosonina E, McCracken S, Li Q, Arnaout R, Mientjes E, Nickerson JA, Awrey D, Greenblatt J, Grosveld G, et al: The acute myeloid leukemia-associated protein, DEK, forms a splicing-dependent interaction with exon-product complexes. J Cell Biol 150: 309-320, 2000.

13. Alexiadis V, Waldmann T, Andersen J, Mann M, Knippers R and Gruss C: The protein encoded by the proto-oncogene DEK changes the topology of chromatin and reduces the efficiency of DNA replication in a chromatin-specific manner. Genes Dev 14: $1308-1312,2000$.
14. Kavanaugh GM, Wise-Draper TM, Morreale RJ, Morrison MA, Gole B, Schwemberger S, Tichy ED, Lu L, Babcock GF, Wells JM, et al: The human DEK oncogene regulates DNA damage response signaling and repair. Nucleic Acids Res 39: 7465-7476, 2011.

15. Kim DW, Chae JI, Kim JY, Pak JH, Koo DB, Bahk YY and Seo SB: Proteomic analysis of apoptosis related proteins regulated by proto-oncogene protein DEK. J Cell Biochem 106: 1048-1059, 2009

16. Lee KS, Kim DW, Kim JY, Choo JK, Yu K and Seo SB: Caspasedependent apoptosis induction by targeted expression of DEK in Drosophila involves histone acetylation inhibition. J Cell Biochem 103: 1283-1293, 2008.

17. Casas S, Nagy B, Elonen E, Aventín A, Larramendy ML, Sierra J, Ruutu T and Knuutila S: Aberrant expression of HOXA9, DEK, CBL and CSF1R in acute myeloid leukemia. Leuk Lymphoma 44: 1935-1941, 2003

18. Lin L, Piao J, Gao W, Piao Y, Jin G, Ma Y, Li J and Lin Z: DEK over expression as an independent biomarker for poor prognosis in colorectal cancer. BMC Cancer 13: 366, 2013.

19. Piao J, Shang Y, Liu S, Piao Y, Cui X, Li Y and Lin Z: High expression of DEK predicts poor prognosis of gastric adenocarcinoma. Diagn Pathol 9: 67, 2014.

20. Ahmad I and Rao DN: Chemistry and biology of DNA methyltransferases. Crit Rev Biochem Mol Biol 31: 361-380, 1996.

21. Gardiner-Garden $\mathrm{M}$ and Frommer $\mathrm{M}: \mathrm{CpG}$ islands in vertebrate genomes. J Mol Biol 196: 261-282, 1987.

22. Cottrell SE: Molecular diagnostic applications of DNA methylation technology. Clin Biochem 37: 595-604, 2004.

23. Stresemann C, Brueckner B, Musch T, Stopper H and Lyko F: Functional diversity of DNA methyltransferase inhibitors in human cancer cell lines. Cancer Res 66: 2794-2800, 2006.

24. Sitwala KV, Adams K and Markovitz DM: YY1 and NF-Y binding sites regulate the transcriptional activity of the dek and dek-can promoter. Oncogene 21: 8862-8870, 2002.

25. Carro MS, Spiga FM, Quarto M, Di Ninni V, Volorio S, Alcalay M and Müller H: DEK Expression is controlled by E2F and deregulated in diverse tumor types. Cell Cycle 5: 1202-1207, 2006.

26. Lü ZL, Luo DZ and Wen JM: Expression and significance of tumor-related genes in HCC. World J Gastroenterol 11: 3850-3854, 2005

27. Lin LJ and Chen LT: The role of DEK protein in hepatocellular carcinoma for progression and prognosis. Pak J Med Sci 29: 778-782, 2013.

28. Yi HC, Liu YL, You P, Pan JS, Zhou JY, Liu ZJ and Zhang ZY: Overexpression of DEK gene is correlated with poor prognosis in hepatocellular carcinoma. Mol Med Rep 11: 1318-1323, 2015.

29. Kondoh N, Wakatsuki T, Ryo A, Hada A, Aihara T, Horiuchi S, Goseki N, Matsubara O, Takenaka K, Shichita M, et al: Identification and characterization of genes associated with human hepatocellular carcinogenesis. Cancer Res 59: 4990-4996, 1999.

30. Roman-Gomez J, Jimenez-Velasco A, Agirre X, Cervantes F, Sanchez J, Garate L, Barrios M, Castillejo JA, Navarro G, Colomer D, et al: Promoter hypomethylation of the LINE-1 retrotransposable elements activates sense/antisense transcription and marks the progression of chronic myeloid leukemia. Oncogene 24: 7213-7223, 2005.

31. Li S, Hansman R, Newbold R, Davis B, McLachlan JA and Barrett JC: Neonatal diethylstilbestrol exposure induces persistent elevation of c-fos expression and hypomethylation in its exon-4 in mouse uterus. Mol Carcinog 38: 78-84, 2003.

32. Dang CV, O'donnell KA and Juopperi T: The great MYC escape in tumorigenesis. Cancer Cell 8: 177-178, 2005.

33. Rodriguez BAT, Weng YI, Liu TM, Zuo T, Hsu PY, Lin $\mathrm{CH}$, Cheng AL, Cui H, Yan PS and Huang TH: Estrogen-mediated epigenetic repression of the imprinted gene cyclin-dependent kinase inhibitor $1 \mathrm{C}$ in breast cancer cells. Carcinogenesis 32 : 812-821, 2011.

34. Sato N, Maitra A, Fukushima N, van Heek NT, Matsubayashi H, Iacobuzio-Donahue CA, Rosty C and Goggins M: Frequent hypomethylation of multiple genes overexpressed in pancreatic ductal adenocarcinoma. Cancer Res 63: 4158-4166, 2003. 